\title{
Evolution in parallel: new insights from a classic system
}

\author{
Susan A. Foster and John A. Baker \\ Department of Biology, Clark University, Worcester, MA 01610, USA
}

\begin{abstract}
Neo-darwinists have long argued that parallel evolution, the repeated evolution of similar phenotypes in closely related lineages, is caused by the action of similar environments on alleles at many loci of small effect. A more controversial possibility is that the genetic architecture of traits initiates parallelism, sometimes through fixation of alleles of large effect. Recent research (by Cole et al., Colosimo et al., Cresko et al., and Shapiro et al.) offers the surprising insight that reduction in two armor traits of threespine stickleback is governed by independently segregating major loci as well as additional quantitative trait loci ( $Q T L)$, and that alleles at the same major loci are associated with parallel phenotypes in globally distributed populations. This research suggests the emergence of a new and exciting vertebrate model system for evolutionary genetics.
\end{abstract}

A lineage that diversifies extensively in response to divergent ecological conditions produces sets of differentiated populations, or higher order taxa, collectively termed an 'adaptive radiation'. Adaptive radiations can comprise many populations and species that have evolved in parallel under similar environmental conditions, strongly implicating natural selection as a cause of the parallel evolution [1,2]. Although convergent patterns of natural selection on quantitative traits can explain this parallelism, alternative mechanisms range from similarly correlated genetic variation biasing evolution [3] to ancestral patterns of phenotypic plasticity that reveal characteristic phenotypes in novel environments, biasing responses to selection [2]. Unfortunately, our understanding of the genetic underpinnings of parallel evolution is often impeded by barriers to reproduction between derived species, precluding the use of genetic crosses to determine the number and locations of causal genetic factors. New research demonstrates how crosses among divergent, compatible populations of the threespine stickleback Gasterosteus aculeatus species complex can elucidate the genetic causes of parallel evolution in an adaptive radiation [4-7].

Fossil remains of oceanic stickleback indicate that they have changed little morphologically during the past 10 million years [8], but have repeatedly given rise to freshwater populations that have differentiated extensively [9]. The most recent freshwater colonization and diversification began $\sim 22000$ years ago as glaciers receded, creating unoccupied freshwater habitats into which populations could expand. Subsequent differen-

Corresponding author: Susan A. Foster (sfoster@clarku.edu).

Available online 28 July 2004 tiation has produced phenotypically parallel populations that have evolved repeatedly and independently in similar habitats $[9,10]$, suggesting a strong adaptive component to the divergence $[1,2,8]$. Remarkably, individuals from geographically disparate and divergent populations can be hybridized in the laboratory using in vitro fertilization techniques, enabling the use of molecular markers to map genes and phenotypes [11].

Throughout their range, oceanic stickleback have bony armor comprising lateral plates and a robust set of dorsal and pelvic spines that serve as defenses against predators (Figure 1a). Upon invasion of freshwater, the posterior lateral plates are typically lost, leaving a few anterior lateral plates that articulate with the pelvic girdle [12] (Figure 1b,c). Loss of the lateral plates can be rapid [13] (Figure 1d), supporting an earlier inference of strong selection against the full complement of lateral plates in freshwater, probably as a result of reduced ion availability and lower predation intensity [12,14].

Crosses between complete and low-plated populations reveal that a major genetic factor governs the number of all but the most anterior lateral plates, and that the completely plated phenotype is specified by a dominant allele [5,6]. The ancestral complete phenotype was not restored in crosses between low-plated populations (among three Alaskan populations [6], and between a British Columbia population crossed with Californian [5] and Japanese [15] populations) as might have occurred if the low-plated phenotypes in different populations were due to mutations in different genes of large effect, enabling genetic complementation in the hybrids. Although the major lateral plate loci are reported to map to different linkage groups $[5,6]$, the published stickleback genetic map has more linkage groups than it does chromosomes [11]. The stn 183 and stn 184 markers, to which the Alaskan mendelian lateral plate locus is tightly linked [6], map with markers linked to the major lateral plate quantitative trait locus (QTL) on LGIV in the British Columbia $\mathrm{x}$ Japanese cross. Together, the genetic mapping and complementation results suggest that the global phenomenon of rapid, parallel lateral plate loss in stickleback has the same simple genetic basis in many different populations.

Loss or reduction of stickleback pelvic armor (Figure 1a,c) is less common and is usually associated with reduced ion availability and the absence of piscine predators [16]. Nevertheless, in stickleback from different geographical regions, a single mendelian factor exerts control over pelvic expression, and the full pelvic girdle is specified by a dominant allele. Pelvic armor loss maps to a different chromosomal region (LGVII) compared 

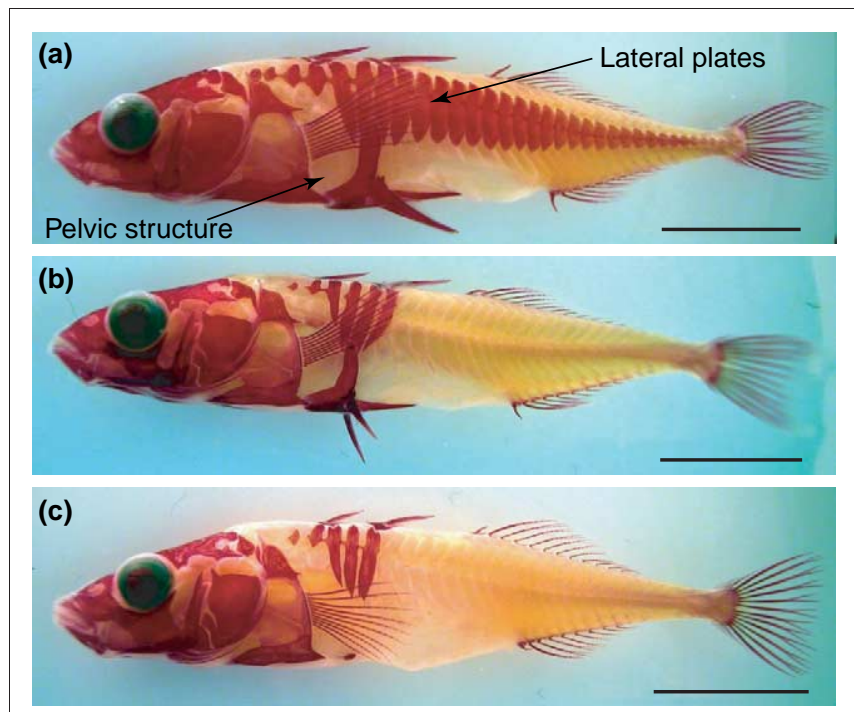

(d)(i)
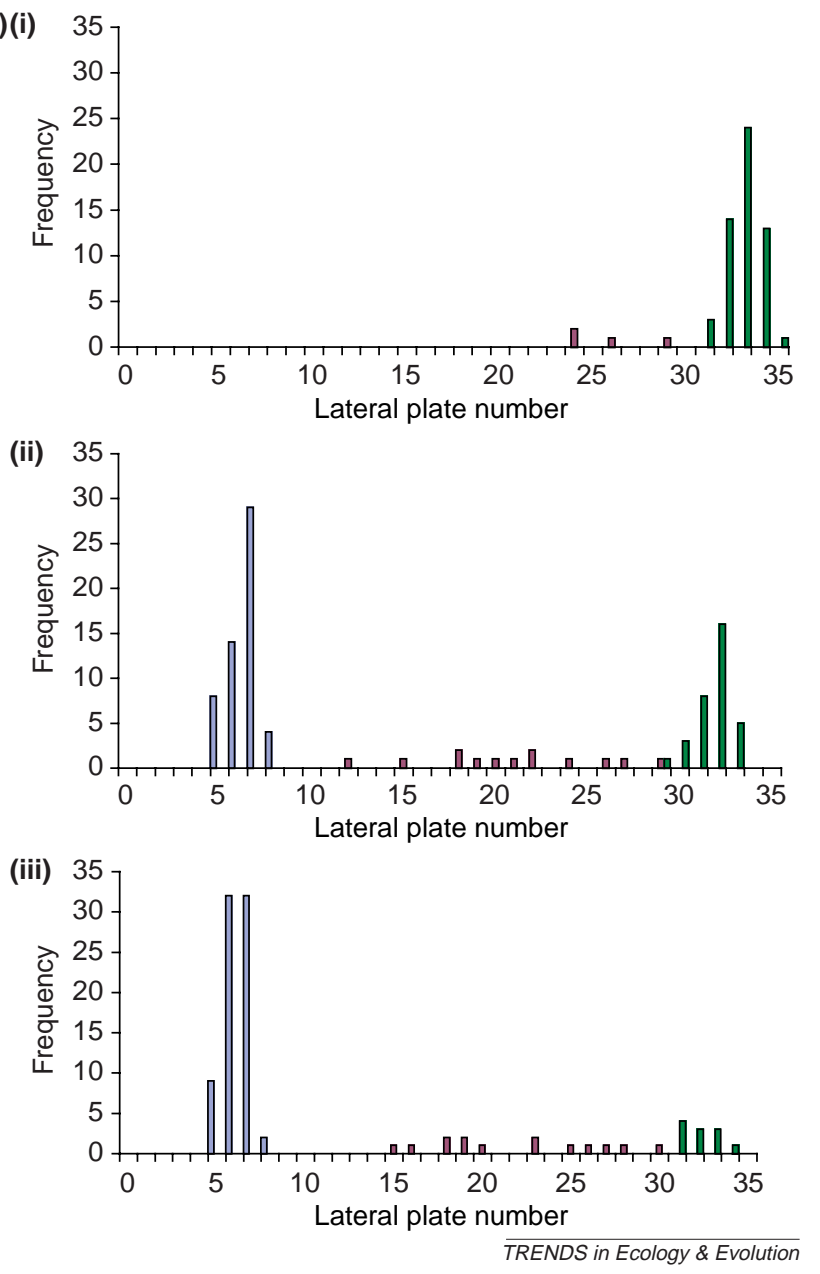

Figure 1. Armor morphology and the high rate of lateral plate loss in a natural population of threespine stickleback. Trypsin-cleared, alizarin red-stained stickleback representative of the fully plated type with a robust pelvic girdle (a), the lowplated form with a functional pelvic girdle that is characteristic of populations in most freshwater habitats (b), and a pelvic-reduced form exhibiting extreme reduction of the pelvic girdle (c). Images reproduced with permission from W.A. Cresko. The histogram series (d) shows the change in frequency of fully plated (green) and low-plated (blue) morphs in Loberg Lake, Alaska from 1990 (di) through 1996 (dii) to 2001 (diii; data taken with permission from [13]; lateral plate numbers from left-hand side only). Purple bars indicate individuals with intermediate numbers of plates. 1990 was the first year that stickleback were captured from Loberg Lake after they had been eradicated with rotenone in 1982. All were apparently anadromous in origin [13]. Scale bar $=10 \mathrm{~mm}$. with lateral plate loss. However, both mapping and complementation crosses suggest that the same locus controls pelvic reduction in geographically disparate populations [6,7].

Pelvic-reduced stickleback display the same directional asymmetry that is seen in pitx1-knockout mice [4,7]. Pitx1 is expressed during the development of several vertebrate structures, including the hind limb. Cole et al. first demonstrated that this transcription factor is expressed during normal pelvic development in stickleback, but not in the pelvic region of spine-deficient stickleback [4,7] (Figure 2). Shapiro et al. demonstrate that Pitx1 maps to the end of LGVII, where the major pelvic reduction locus also resides, and that it falls within the region of highest probability for the major pelvic reduction QTL [7]. Finding no sequence differences in the pitx 1 coding regions of full compared with reduced pelvic structure stickleback, the authors conclude that cis-regulatory changes in pitx 1 cause the loss of pelvic structure in freshwater habitats. However, family sizes are small, and no gene on the distal $10 \%$ of the chromosome is ruled out. As genes affecting the same trait are often clustered, a role for other closely linked genes cannot yet be excluded [4]. Nevertheless, involvement of pitx 1 and other genes ( $t b x 4$, pitx 2 , hox and $f g f s$ ) in stickleback pelvic development offers crucial evidence that the developmental pathways and genes uncovered in mutagenesis studies can be involved in evolutionary change in wild populations [4,7].

Results of QTL mapping [5,7,11] and complementation crosses between individuals from low-armor populations [6] indicate that minor loci also contribute to armor variation, an inference supported by quantitative genetic analyses of armor variation in stickleback [17-19]. These minor loci can, but do not always, map to the same locations in different populations [5,7]. Furthermore, differences in the degree of complementation between pairs of armor-reduced Alaskan populations suggest different distributions of variants at minor loci [6]. Thus, freshwater populations might have retained different subsets of the genetic variation present in oceanic populations at minor loci, as has been shown for neutral loci (W.A. Cresko, PhD thesis, Clark University, 2000) [10].

The parallel evolution that is apparent in these studies seems to involve the same genetic factors of large effect within geographical regions (Alaska), along the edge of an ocean basin (west coast of North America) and between the Pacific and Atlantic basins. A rich literature, stimulated by the early behavioral research of Tinbergen [20], and the ensuing efforts of D.W. Hagen and J.D. McPhail [9] offers evidence of similar stickleback population variation in many features that also are likely to reflect adaptive genetic differentiation. Among these are body shape differences, including the well characterized benthiclimnetic distinctions, an array of behavioral and lifehistory phenotypes, and color variation [9,21-24]. The stickleback radiation also offers an opportunity to explore the genetics of speciation because sympatric species pairs have evolved repeatedly and in parallel, and can be hybridized [1,10,22,24]. Much remains to be explored in this remarkable radiation and, as these new studies demonstrate, the tools are now becoming available to 

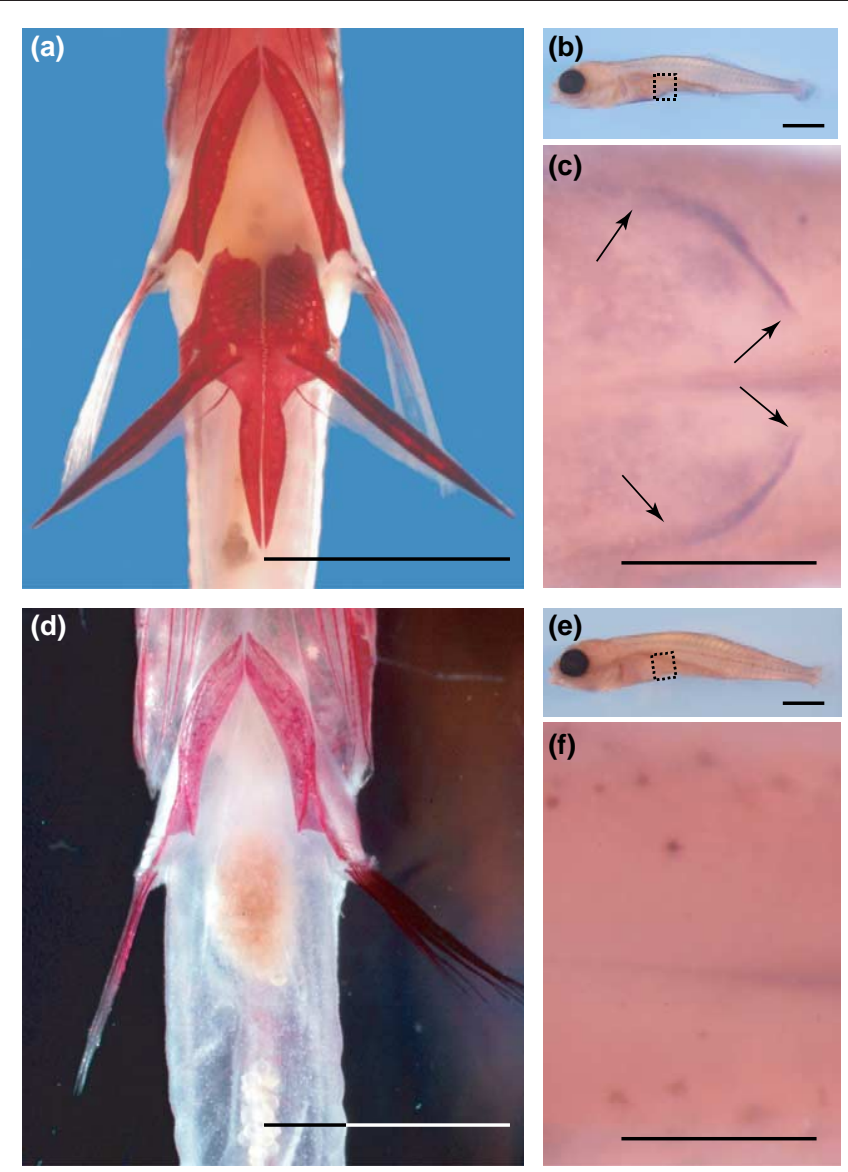

$\overline{T R E N D S}$ in Ecology \& Evolution

Figure 2. Altered pattern of Pitx 1 expression during larval development of stickleback from pelvic-reduced populations. Fish from populations with robust pelvic structures [(a) juvenile fish, ventral view] show clear expression of Pitx1 in the hind fin region [indicated by arrows in (c)] during larval development $[(\mathbf{b}, \mathbf{c})$ stage 30 larvae, side and ventral view]. By contrast, strongly pelvic-reduced fish from Paxton Lake, British Columbia (d) do not express Pitx 1 in the prospective hind fin region [(e,f) stage 30 larvae], and develop into adult fish that lack both the bony pelvis and the large projecting pelvic spines. Modified with permission from [7] Additional images reproduced with permission from M. Shapiro and D. Kingsley.

add the evolution of developmental programs to the range of evolutionary research conducted with stickleback.

The development of stickleback into a mature model for evolutionary genetics requires substantial work. Significant progress has already been made as a result of contributions from laboratories involved in research on other model systems. The University of Oregon group (http://neuro.uoregon.edu/ionmain/htdocs/faculty/ postleth.html) developed many of the embryonic techniques used with zebrafish (http://zfin.org) [6], and is a center of study of fish genome evolution [25]. Similarly, expertise in the embryology of limb formation and loss in other vertebrates informed research on stickleback pelvic reduction at the University of Dundee (http:// www.dundee.ac.uk/biocentre/SLSBDIV4cat.htm) $[4,26]$. Finally, the Stanford group (http://kingsley.stanford. edu) [5,7] has applied to stickleback techniques that were developed for research on skeletal development in mice [27]. These and other laboratories have developed methods for the analysis of gene expression during development $[4,7,28,29]$, for generating transgenic stickleback [30], and have provided the first genomewide linkage map for stickleback [11].

Recognizing the increasing importance of the stickleback model system, the NIH established a Center of Excellence in Genomic Sciences (CEGS) at Stanford University in 2002 to develop genomic resources for fish. The CEGS has already produced large EST collections, bacterial artificial chromosome libraries and an initial physical map of the stickleback genome, all of which are publicly available [27] (http://www.cegs.stanford.edu/ index.jsp). An annual workshop is offered at Stanford that presents research techniques for this emerging model organism and, in January 2004, the NIH approved the stickleback for high-priority genome-wide sequencing (http//www.genome.gov/10002154). Thus, we will soon have in place an array of tools that is similar to those available for other model organisms.

Stickleback could serve as a model system for investigating human disease and classic problems in evolution. There are many human analogues for the loss of bone and cartilage, and for changes in behavior and coloration. From an evolutionary perspective, the pronounced parallelism in this radiation could be due to an ancestral polymorphism in the oceanic form, or to a recurrent biased mutation [15]. However, this emphasis on genetic determination should not obscure the role that variation in minor loci, interacting with environmental conditions, could play in revealing phenotypic variation when stickleback invade novel freshwater habitats [3]. This, and other causes of phenotypic plasticity at the initiation of adaptive radiation, could influence the patterns of phenotype divergence [6]. The appeal of the threespine stickleback adaptive radiation to population biologists, developmental biologists and geneticists is that these, and many other questions can be explored using this species complex.

\section{References}

1 Schluter, D. (2000) The Ecology of Adaptive Radiation, Oxford University Press

2 West Eberhard, M.J. (2003) Developmental Plasticity and Evolution, Oxford University Press

3 Haldane, J.B.S. (1932) The Causes of Evolution, Longmans and Green

4 Cole, N.J. et al. (2003) Expression of limb initiation genes and clues to the morphological diversification of threespine stickleback. Curr. Biol. 13, R951-R952

5 Colosimo, P.F. et al. (2004) The genetic architecture of parallel armor plate reduction in threespine sticklebacks. PLoS Biol. 2, 635-641

6 Cresko, W.A. et al. (2004) Parallel genetic basis for repeated evolution of armor loss in Alaskan threespine stickleback populations. Proc. Natl. Acad. Sci. U. S. A. 101, 6050-6055

7 Shapiro, M.D. et al. (2004) Genetic and developmental basis of evolutionary pelvic reduction in threespine sticklebacks. Nature 428 , $717-723$

8 Bell, M.A. (1994) Paleobiology and evolution of threespine stickleback. In The Evolutionary Biology of the Threespine Stickleback (Bell, M.A. and Foster, S.A., eds), pp. 438-471, Oxford University Press

9 Bell, M.A. and Foster, S.A. (1994) Introduction to the evolutionary biology of the threespine stickleback. In The Evolutionary Biology of the Threespine Stickleback (Bell, M.A. and Foster, S.A., eds), pp. 1-27, Oxford University Press

10 Taylor, E.B. and McPhail, J.B. (2000) Historical contingency and determinism interact to prime speciation in sticklebacks Gasterosteus. Proc. R. Soc. Lond. Ser. B 267, 2375-2384 
11 Peichel, C.L. et al. (2001) The genetic architecture of divergence between threespine stickleback species. Nature 414, 901-905

12 Reimchen, T.E. (1994) Predators and morphological evolution in threespine stickleback. In Evolutionary Biology of the Threespine Stickleback (Bell, M.A. and Foster, S.A., eds), pp. 240-276, Oxford University Press

13 Bell, M.A. et al. (2004) Twelve years of contemporary armor evolution in a threespine stickleback population. Evolution 58, 814-824

14 Hagen, D.W. and Gilbertson, L.G. (1973) Selective predation and the intensity of selection acting upon the lateral plates of threespine sticklebacks. Heredity 30, 273-287

15 Schluter, D. et al. (2004) Parallel evolution and inheritance of quantitative traits. Am. Nat. 163, 809-822

16 Bell, M.A. et al. (1994) Evolution of pelvic reduction in threespine stickleback fish: a test of competing hypotheses. Evolution 47, 906-914

17 Hagen, D.W. and Gilbertson, L.G. (1973) The genetics of plate morphs in freshwater threespine sticklebacks. Heredity 31, 75-84

18 Hatfield, T. (1997) Genetic divergence in adaptive characters between sympatric species of stickleback. Am. Nat. 149, 1009-1029

19 Hermida, M. et al. (2002) Heritability and 'evolvability' of meristic characters in a natural population of Gasterosteus aculeatus. Can. J. Zool. 80, 532-541

20 Tinbergen, N. (1951) The Study of Instinct, Oxford University Press

21 Baker, J.A. (1994) Life history variation in female threespine stickleback. In The Evolutionary Biology of the Threespine Stickleback (Bell, M.A. and Foster, S.A., eds), pp. 144-187, Oxford University Press
22 McPhail, J.D. (1994) Speciation and the evolution of reproductive isolation in the sticklebacks (Gasterosteus): origin of sympatric pairs. In The Evolutionary Biology of the Threespine Stickleback (Bell, M.A. and Foster, S.A., eds), pp. 399-347, Oxford University Press

23 Foster, S.A. et al. (1997) Patterns of homoplasy in behavioral evolution. In Homoplasy and the Evolutionary Process (Sanderson, M.J. and Hufford, L., eds), pp. 245-269, Academic Press

24 McKinnon, J.S. and Rundle, H.D. (2002) Speciation in nature: the threespine stickleback model systems. Trends Ecol. Evol. 17, 480-488

25 Amores, A.A. et al. (1998) Zebrafish hox clusters and vertebrate genome evolution. Science 282, 1711-1714

26 Tanaka, M. et al. (2002) Fin development in a cartilaginous fish and the origin of vertebrate limbs. Nature 416, 527-531

27 Kingsley, D.M. et al. New genomic tools for molecular studies of evolutionary change in stickleback. Behaviour (in press)

28 Ahn, D. and Gibson, G. (1999) Axial variation in the threespine stickleback: relationship to Hox gene expression. Dev. Genes Evol. 209, $473-481$

29 Cresko, W.A. et al. (2003) Genome duplication, subfunction partitioning, and lineage divergence: Sox9 in stickleback and zebrafish. Dev. Dyn. 228, 480-489

30 Hosemann, K.E. et al. (2004) A simple and efficient microinjection protocol for making transgenic sticklebacks. Behaviour (in press)

\title{
Pesticides, mortality and population growth rate
}

\author{
Benedikt R. Schmidt ${ }^{1,2}$
}

\author{
${ }^{1}$ Zoologisches Institut, Universität Zürich, Winterthurerstrasse 190, 8057 Zürich, Switzerland \\ ${ }^{2} \mathrm{KARCH}$, Naturhistorisches Museum, Bernastrasse 15, 3005 Bern, Switzerland
}

In a recent article in TREE, Sih and colleagues point out that standard ecotoxicology tests might not be useful for assessing the real threat from a pesticide for non-target organisms [1]. Pesticide effects might be seriously underestimated if their interactive effects with other stressors, such as predators, are ignored [2,3]. This insight is important and will help to improve ecotoxicology tests. Yet, ecotoxicology testing, regardless of whether interactive effects are included, still has a major shortcoming.

Typical ecotoxicology tests examine the effects of pesticides on single vital rates, usually mortality. Such individual-level responses are relatively easy to study and can provide important first insight. However, they do not inform us about whether the population as a whole will suffer [4]; from a conservation perspective, it is these population-level effects that matter [4]. Thus, ecotoxicology tests should be done at the population level, unless it can be shown that individual-level responses are good proxies for population-level responses. Responses of individuals might inform about population performance when mortality is very high. Often, however, mortality is intermediate and there can be sub-lethal effects [5]. As pointed out by Forbes and Calow, three questions need to

Corresponding author: Benedikt R. Schmidt (bschmidt@zool.unizh.ch).

Available online 15 July 2004 be answered before the population-level effects of toxicants can be assessed [4]: (i) to what extent do individual-level variables underestimate or overestimate population-level responses?; (ii) how do toxicant-caused changes in individual-level variables translate into changes in population dynamics?; and (iii) to what extent are the population-level effects influenced by density-dependent (compensatory) responses? Studying the population-level effects of toxicants is a challenging task, but the experimental study by Forbes and colleagues shows that it can be done [6].

The link between individual-level and population-level responses is poorly understood in amphibians with complex life cycles $[7,8]$. Sih and colleagues discuss experiments carried out with larval amphibians, for which mortality was the response variable [2,3]. Population models suggest that the increased mortality in the tadpole stage has relatively minor effects on population growth $[7,8]$, because density dependence is often strong in the tadpole stage [7]. Generally, terrestrial stages appear to be more important for the maintenance of positive population growth [7,8]. Although large-scale negative effects of pesticide use on amphibian populations have been shown in correlational studies [9], the work described by Sih and colleagues might help us to understand only a little about the processes leading to local and global 